\title{
The Effects of Sensory Flow on The Autonomic Nervous System
}

\author{
Jessica Keller ${ }^{1 *}$, Scott Musgrave ${ }^{2}$, Alan Nasypany ${ }^{3}$, James May ${ }^{4}$ and Adam Thompson ${ }^{5}$ \\ ${ }^{1}$ DAT, Indiana Wesleyan University, USA \\ ${ }^{2} P T$
}

${ }^{3} E d D$, University of Idaho, USA

${ }^{4} D A T$, University of Idaho, USA

${ }^{5}$ Ph.D., Indiana Wesleyan University, USA

*Corresponding author: Jessica Keller, Department of Health and HumanPerformance, USA.

Received Date: September 12, 2019

Published Date: September 26, 2019

\begin{abstract}
Treating patients with chronic pain often proves difficult for medical professionals. One potential contribution to chronic pain is that upregulation of the autonomic nervous system (ANS) may cause the body to enter faulty pain cycles. Interrupting ANS up-regulation may break these faulty pain cycles. Sensory Flow is a manual therapy technique thought to interrupt ANS up-regulation. Twenty patients volunteered and were randomly assigned to either a group receiving Sensory Flow or a control group. Results indicate Sensory Flow significantly improved pain $(p<$ .004 , partial $\left.\eta_{2}=.439\right)$ and stress perception $\left(p=.020\right.$, partial $\left.\eta_{2}=.169\right)$ following the second treatment. Therefore, Sensory Flow may be useful in breaking faulty pain cycles in patients who are up regulated.
\end{abstract}

\section{Background}

Treating patients who have chronic pain is often challenging for health care professionals. Typically, patients with chronic pain present with a variety of comorbidities, including, but not limited to decreased range of motion, muscle spasm, and recurring muscle tenderness [1]. Many of these protective patterns have also been observed as components of the fight, flight or freeze response. An overactive fight, flight or freeze response, a product of the Autonomic Nervous System (ANS), has been shown to be a major contributor to chronic pain response patterns and persistent pain [1-3]. One fight, flight or freeze theory that is starting to gain momentum is explained well in Bessel van der Kolk's book, The Body Keeps the Score: Brain, Mind and Body in the Healing of Trauma [4]. Van der Kolk explains that if the person having a stressful experience successfully completes the fight or flight cycle, the body recognizing that the threat has passed, will allow the stress of the trauma to dissipate. If the brain chooses fight or flight, but the body is unsuccessful, it enters a freeze cycle. During the freeze cycle the body becomes immobile. Often, predators become less interested in prey that is not moving, which allows the prey to escape. Successful

evasion of a threat prepares the body to discharge trauma. If a body never experiences a discharge, the cycle is incomplete, and the stress is stored in the brain as procedural memory [4].

The stored input tells the brain how to react every time a similar sensory input or memory a rises [4,5]. An important thing to note is every detail about the event is stored [2,5]. Stimuli like scents, lighting, noises, sensations and objects become part of the sensitizing factors. These stored stimuli are called kindling [5]. While kindling often strengthens the original traumatic memory, it can also branch out into new pathways. When kindling recurs in everyday life, the amygdala prepares the body by activating the Sympathetic Nervous System (SNS) for fight or flight $[3,4,6]$. When the fight, flight, or freeze cycle completes properly, the threat to the body is extinguished, sensory information is stored, and the body returns to its normal set point. When it does not complete the cycle properly, the amygdala continues to seek resolution. The incomplete cycle results in chronic up-regulation of the ANS [3,5,7-10].

The purpose of the ANS is to balance internal functions in the body. The ANS is composed of two opposing divisions: The 
Sympathetic and Para Sympathetic Nervous Systems (PSNS). When the ANS is functioning properly the body will transition smoothly between sympathetically and parasympathetically mediated states $[11,12]$. When the ANS is up-regulated, however, the cycling becomes rapid and asynchronous [13,14]. This creates confusion in even the most basic bodily functions, including breathing and heart rate [15-17]. Up-regulation of the ANS can also cause activation of faulty pain cycles [18]. Stress and a perceived immediate threat to the body can cause the body to feel less pain, but prolonged stress can make the body more susceptible to feeling pain [19]. Memories, anxiety or re-experiencing of stressful events are also capable of eliciting a stress response from the body [20]. The re- experiencing, if it happens often, can drive the ANS into a chronically up-regulated state $[7,8,21,22]$. Sensory Flow is a manual therapy technique designed to stimulate the ANS; however, because there is no threat to the body, the fight, flight or freeze response is not activated.

The practice of Sensory Flow is an "intense repetitive and focused proprioceptive tactile input sufficient to induce a trancelike state, which may serve the purpose of producing extinction of procedural memory patterns and reflex postural motor patterns" [23]. More simply put, Sensory Flow inundates the ANS with safe sensory input that allows the body to complete the fight, flight, or freeze cycle. In Sensory Flow, ANS stimulation is initiated through a predetermined series of touch, which cause mechanoreceptors in the skin to communicate with the SNS. The sensory information that the mechanoreceptors are receiving is perceived to be safe and thus they communicate that there is no present threat of harm to the body [24].

The information allows the balance of power to shift from the SNS to the PSNS [25]. Another theory that might explain this shift of power is known as the Polyvagal theory. The Polyvagal theory explains why constant up-regulation prevents the body from down regulating [26]. The Vagus nerve is divided in to three parts and is responsible for regulation of the ANS. Those divisions are the primitive unmylenated visceral Vagus, which is responsible for digestion and responds to threats by decreasing metabolism, the vagal brake, which inhibits the SNS by slowing down the heart, and the Social Engagement System, which aids in socialization. When one part is heavily recruited, the other two are not easily accessible [26]. If the body is up-regulated, the vagal brake and social engagement systems would be more difficult to access, thus impeding down-regulation [26,27]. Autonomic Nervous System upregulation happens for a variety of reasons, but some of the most common are persistent stress or anxiety states, often caused by an insufficient resolution of a fight, flight or freeze response [3].

Table 1:

\begin{tabular}{|c|c|c|}
\hline Outcome Measure & $\begin{array}{r}\text { Explanation } \\
\text { Stress-O-Meter }\end{array}$ & $\begin{array}{r}\text { Current stress is rated on a 0-10 interval scale, with 0 representing no stress and 10 representing the highest amount of } \\
\text { stress the participant can imagine. While there is no published data on the validity or reliability of this particular scale, it is } \\
\text { considered an interval scale, and can adequately provide data for parametric analysis [48]. }\end{array}$ \\
\hline Heart Rate Variability & $\begin{array}{r}\text { Assessing HRV, specifically coherence, will allow us to measure the level of activation of the ANS, and to know which part, SNS } \\
\text { or PSNS, to attribute the activation of the ANS to. We know this because the greater the coherence the greater the activation of } \\
\text { the PSNS. The converse is also true, in that if coherence does not change, the PSNS was not greatly affected [49]. }\end{array}$ \\
\hline
\end{tabular}

Perception of the initial painful experience and kindling associated with that event could cause misinterpretation of the pain response altogether. Identifying potential therapeutic interventions could prove an invaluable resource in treating patients suffering from chronic pain. The purpose of this study was to explore the effects of Sensory Flow on the ANS as measured by changes in blood pressure (BP), pulse, heart rate variability (HRV), perceived stress, and muscle tenderness following treatment, which are standard measures used in assessing the ANS [28-30].

\section{Methods}

\section{Design}

Randomized, blinded, repeated measure study comparing a treatment group who received Sensory Flow, with a control group who rested quietly on a table for 15 minutes.

\section{Setting}

Department of Health and Human Performance, Indiana Wesleyan University, USA.

\section{Sample}

Twenty participants (16 females, 4 males) were recruited for the study from a small private college.

\section{Inclusion}

All participants were: 1) between 18 and 25 years of age, 2) had a score on the Stress Test of greater than 150 Holmes T \& Rahe $\mathrm{R}$ [31] 3) had a significant score for any of the three components of the Depression, Anxiety and Stress Scale Henry JD \& Crawford JR [32] (DAS scale), and 4) had not consumed caffeine three hours prior to the study [33].

\section{Exclusion criteria}

Patients were excluded if they 1) did not sign the consent and HIPAA forms, 2) had skin infection, 3) had skin hypersensitivity that would prevent them from being touched, or 4) their schedule did not allow protocol to be followed completely. Protocol for the study was approved by both the University of Idaho, and Indiana Wesleyan University Institutional Review Boards.

\section{Instrumentation}

The Stress test and the DAS Scale were used as inclusion criteria. The STRESS-O-METER, BP, Pulse, HRV, and Sympathetic Activation Pattern (SAP) measurements were taken before and after treatment at both appointments. For a more detailed description of each outcomes measure see Table 1. 


\begin{tabular}{|c|c|}
\hline $\begin{array}{c}\text { Sympathetic Activation } \\
\text { Pattern }\end{array}$ & $\begin{array}{c}\text { The SAP is used to evaluate the tension, tenderness and reactivity of nine predetermined muscular regions associated with } \\
\text { the startle reflex and up-regulation of the ANS [23]. }\end{array}$ \\
\hline $\begin{array}{c}\text { Depression, Anxiety, } \\
\text { and Stress Scale }\end{array}$ & $\begin{array}{c}\text { 42-item self-report survey designed to measure depression, anxiety and stress. In the scale each item is weighted towards one } \\
\text { of three categories: depression, anxiety or stress [50]. Scores are considered significant when above } 9 \text { for depression, 7 for } \\
\text { anxiety and 14 for stress. The DAS scale was designed to measure severity of symptoms, and not to detect significant changes } \\
\text { in depression, anxiety or stress [50]. The DAS scale has been shown to possess adequate construct validity. Reliability for this } \\
\text { scale was measured at .88 for depression, .82 for anxiety, .90 for stress, and .93 for the total scale [32]. }\end{array}$ \\
\hline Stress Test & $\begin{array}{r}\text { A list of stressors complied by Thomas H. Homes and Richard H Rahe, in 1967. The Stress Test was designed to provide a } \\
\text { standard of measure for the impact of a wide range of common stressors. Interpretation of the scale is difficult because it does } \\
\text { not take into account coping mechanisms of each individual. A total of less than 150 is considered a low level of stress [31]. }\end{array}$ \\
\hline
\end{tabular}

\section{Procedures}

The primary investigator (PI) and research assistants recruited participants from a private NAIA university. Slottr (Slottr.com, Vancouver, British Columbia, Canada), a sign-up website, was used to schedule appointments. Each participant was contacted by email before their appointment, reminded of their scheduled time, and asked not to consume caffeine for three hours prior. All participants were asked to wear a tank top and shorts. Female participants were asked to wear a sports bra, and male participants were asked to remove their shirts, to ensure adequate access for palpation. When participants arrived, the researchers confirmed the participants had followed the preparation procedures. If the procedures had been followed, the participant completed the Stress Test and the DAS scale. Both scales were scored to ensure that the participant met the inclusion criteria. If participants did not follow the preparation procedures they were asked to reschedule the appointment. After completing the consent forms and questionnaires, participants were given a physical examination, were assigned to their treatment group, and experienced their first round of treatment.

\section{Physical exam}

The physical exam was a standard musculoskeletal and orthopedic physical examination. The exam began with blood pressure assessment using an Omron M6 HEM-7001-E Upper Arm Blood Pressure Monitor with Comfit Cuff (Omron Healthcare, Lake Forest, IL). The Omron M6 blood pressure cuff was deemed in accordance with International Protocol criteria for use by adults, the elderly, and the obese $[34,35]$. The participant was placed in a supine position with arm resting next to the participant on the plinth. The researcher used the participants left arm to obtain all blood pressure measurements.

Participants then had their pulse taken using a SantaMedical 110 pulse oximeter, (Beijing, Choice Electronic Company, Beijing, China). The participant lay supine with hand resting comfortably on the plinth. The researcher used the participant's right index finger for all pulse measurements. The Sympathetic Activation Pattern (SAP) muscles were palpated for tenderness. The SAP was used to evaluate the tension, tenderness and reactivity of nine predetermined muscular regions associated with ANS upregulation (Table 2) $[23,36,37]$. The area of most tenderness of each muscle was marked with an ultraviolet marker to ensure accurate measures were taken post treatment. The most tender area was tested with predetermined pressure from a dolorimeter (Table 2). A dolorimeter is a force gauge with a rubber disc surface of $1 \mathrm{~cm} 2$. The gauge is calibrated in $\mathrm{kg} / \mathrm{cm} 2$ [38]. When the dolorimeter reading was equal to the standard pressure from that structure the measurement was recorded and the participant was asked to rate their pain on a 0-10 numeric rating scale (NRS). If a participant believed the pressure to be too great while attempting to reach the predetermined pressure, they were instructed to say stop, and the tolerated pressure was noted. Jump signs or attempts to withdraw from the pressure were also noted. The measurements were taken following a standard protocol that assures validity and reliability [38]. After completing the physical exam, participants were randomly assigned in equal number to either the treatment group or the control group. The control group did not receive Sensory Flow treatment but completed all of the same preliminary measurements as the treatment group.

Table 2: List of Sympathetic Activation Pattern muscles and the standardized dolorimeter pressure 468 used for each.

\begin{tabular}{|c|c|c|}
\hline $\begin{array}{l}\text { Sympathetic Activation } \\
\text { Pattern Muscles }\end{array}$ & $\begin{array}{l}\text { Dolorimeter } \\
\text { Suggestion }\end{array}$ & $\begin{array}{l}\text { Average (If } \\
\text { necessary) }\end{array}$ \\
\hline Gastrocnemius & $6.0 \mathrm{~kg} / \mathrm{cm}^{2}[51]$ & \\
\hline Hip Adductors & $6.0 \mathrm{~kg} / \mathrm{cm}^{2}[51]$ & \\
\hline Rectus Femoris & $5.5 \mathrm{~kg} / \mathrm{cm}^{2}[51]$ & \\
\hline Quadratus Lumborum & $4.5 \mathrm{~kg} / \mathrm{cm}^{2}[52]$ & \\
\hline $\begin{array}{l}\text { Thoraco-Lumbar } \\
\text { Paraspinals }\end{array}$ & $\begin{array}{c}\text { Females } 6.1 \text { males } \\
8.8[38]\end{array}$ & $\begin{array}{c}7.5 \mathrm{~kg} / \mathrm{cm}^{2} 2 \mathrm{~cm} \text { from } \\
\text { spine }\end{array}$ \\
\hline Pectoralis Minor & $3.5 \mathrm{~kg} / \mathrm{cm}^{2}$ & \\
\hline Upper Trapezius & $\begin{array}{c}\text { Females } 3.7 \mathrm{~kg} \text { males } \\
5.4 \mathrm{~kg}[38]\end{array}$ & $4.5 \mathrm{~kg} / \mathrm{cm}^{2}$ \\
\hline Masseter & $2.3 \mathrm{~kg} / \mathrm{cm}^{2}[53]$ & \\
\hline
\end{tabular}

\section{Intervention procedures}

Table 3: Timeframes for measurements during treatment 473.

\begin{tabular}{|c|c|}
\hline Time Frame & Measurement \\
\hline Before Treatment & $\begin{array}{c}\text { Hooked up to Heart Rate Variability (HRV), } \\
\text { rest for } 5 \text { minutes }\end{array}$ \\
\hline 2 minutes after treatment & $\begin{array}{c}\text { Blood pressure (BP), pulse, Stress-0-Meter, } \\
\text { HRV }\end{array}$ \\
\hline 8 minutes after treatment & BP, pulse, HRV \\
\hline 14 minutes after treatment & $\begin{array}{c}\text { BP, pulse, HRV, Sympathetic Activation } \\
\text { Pattern }\end{array}$ \\
\hline
\end{tabular}

After group assignment, each participant was connected to the HRV monitor. The participant was allowed to lie in a comfortable position for two minutes while the HRV program calibrated. After calibration and recording of HRV, the participant received their assigned intervention. The intervention lasted approximately 15 minutes. At the conclusion of the assigned intervention, the participant rested quietly for two minutes. After two minutes 
of rest, BP, pulse, and STRESS-O-METER were re-administered. The participant's BP, pulse and HRV were again measured eight and 14 minutes after treatment (Table 3). The increments of BP measurement were chosen based on pilot data conducted by the PI, which indicated that blood pressure taken every 2 minutes after Sensory Flow treatment fell to the lowest measured point at eight minutes, and returned to baseline at an average of 14 minutes. The SAP was then re-palpated, and the participants were again asked to assign a value from 0-10 tenderness, to the marked point. On the second day post-intervention, the participant returned to the clinic for re-evaluation. The participant's BP, pulse, and SAP were taken. The participants also completed the STRESS-O-METER. Following the completion of new measurements, participants again received their assigned intervention. At the conclusion of the intervention, the participants completed the same post-treatment measurement procedure completed after the first intervention session.

\section{Statistical Analysis}

All data were analyzed using SPSS version 22.0 (SPSS Inc. Chicago, IL, USA). G*Power testing using an effect size of $\mathrm{f}=$ 0.5 , and $80 \%$ power Cohen J [39] indicated a sample size of 19 participants. A repeated measure analysis of variance (RM-ANOVA) was conducted for each treatment session to assess the impact of the Sensory Flow verses the control on BP, Pulse, HRV, SOM, and SAP. Blood pressure measurements were converted to a value for mean arterial pressure (MAP) as suggested by The Seventh Report of the Joint National Committee on Prevention, Detection, Evaluation, and Treatment of High Blood Pressure [40]. Significant results were further analyzed using both Bonferroni and Tukey post hoc testing. Prior to data analysis, testing was done to ensure that assumptions of sphericity and normal distribution were met. Effect size was computed with eta squared $\left(\eta_{2}\right)$. Percent change was also calculated on SOM. Percent change was used to determine if the changed in the SOM scores were clinically meaningful to the patient. Minimal Clinically Important Difference for the SOM was determined by Farrar to be 30\% [41]. The formula used to calculate percent change for each group was mean baseline minus mean final score divided by baseline $\left[\left(\left(\mathrm{x}_{2}-\mathrm{x}_{1}\right) / \mathrm{x}_{1}\right) \times 100\right]$.

\section{Results}

Table 4: Characteristics of subjects $(n=20)$.

\begin{tabular}{|c|c|c|}
\hline Characteristics & $\mathbf{n}(\%)$ & Mean \pm SD (min - max) \\
\hline Gender (females) & $16(80)$ & \\
\hline Credit Hours & & $15.36 \pm 1.7(13-17)$ \\
\hline GPA & & $3.45 \pm 0.30(2.8-3.94)$ \\
\hline Year at University & & \\
\hline $1^{\text {st }}$ & $3(15)$ & \\
\hline $2^{\text {nd }}$ & $5(25)$ & \\
\hline $3^{\text {rd }}$ & $8(40)$ & \\
\hline $4^{\text {th }}$ & $4(20)$ & \\
\hline
\end{tabular}

\section{SD: Standard Deviation}

Twenty patients were recruited for the study. (Table 4) All twenty were full time undergraduate students (3 freshmen, 5 sophomores, 8 juniors, 4 seniors). The participants had a mean GPA of 3.45 and were enrolled in 13-17 credit hours ( $\bar{x}=15.36$, SD = 1.7). All of the participants met inclusion criteria on the Stress Test $(\bar{x}=447.25, S D=230.978)$. Control scores on the Stress Test were higher $(M=1070.90, S D=250.83)$ than the treatment group $(M=850.10, S D=244.210)$. There was homogeneity of variances, as assessed by Levene's test for equality of variances ( $p .=.717)$.

\section{Mean arterial pressure}

The change in mean arterial pressure was not statistically significant between groups for treatment one $[F(3,54)=.396$, $p=.686$, partial $\left.\eta_{2}=.022\right]$ or treatment two $[\mathrm{F}(3,54)=3.612, p$ $=.062$, partial $\left.\eta_{2}=.167\right]$. There was significant change over time during treatment one $\left[\mathrm{F}(3,54)=6.947, p=.002\right.$, partial $\left.\eta_{2}=.554\right]$ and but not for treatment $\mathrm{t}(3,54)=1.679, p=.211$, partial $\eta_{2}$ Pulse Changes in pulse were not statistically significant between groups for treatment one $\left[F(3,54)=.262, p=.726\right.$, partial $\left.\eta_{2}=.014\right]$ or treatment two $\left[\mathrm{F}(3,54)=.971, p=.413\right.$, partial $\left.\eta_{2}=.051\right]$. Changes were also significant across time for treatment one $[F(3,54)=$ 5.281, $p=.015$, partial $\left.\eta_{2}=.227\right]$, and for treatment two $[\mathrm{F}(3,54)$ $=4.335 p=.008$, partial $\left.\eta_{2}=.194\right]$.

\section{Heart rate variability}

Heart rate variability was not statistically significant between groups after treatment one $[\mathrm{F}(3,54)=.405, p=.750$, partial $\left.\eta_{2}=.022\right]$ or following treatment two $[\mathrm{F}(3,54)=.839, p=.478$, partial $\left.\eta_{2}=.045\right]$. Changes were also not significant across time for treatments' one $\left[\mathrm{F}(3,54)=.549, p=.621\right.$, partial $\left.\eta_{2}=.032\right]$ or two $\left[\mathrm{F}(3,54)=.365, p=.779\right.$, partial $\left.\eta_{2}=.020\right]$.

\section{Stress-0-meter}

Stress-0-Meter was not statistically significant between groups after treatment one $\left[\mathrm{F}(3,54)=.177, p=.845\right.$, partial $\eta_{2}=$ $.010]$ but was significant after treatment two $[F(3,54)=2.702$, $p=.020$, partial $\left.\eta_{2}=.169\right]$ with a large effect size. The SOM was also significant across time for treatment one $[F(3,54)=41.133$, $p<.000$, partial $\left.\eta_{2}=.696\right]$ and treatmenttwo $[F(3,54)=27.675, p$ $<.000$, partial $\left.\eta_{2}=.650\right]$ with a large effect size.Participants also reported MCID on the Stress-0-Meter ( $>30 \%$ change)(Farrar et al., 2001) in both the Sensory Flow and Control groups in session one, but only for the Sensory Flow group in session two (Figure 1). The Sensory flow group STRESS-O-METER score changed by an average of $39.48 \%$ during treatment one and by $45.65 \%$ during treatment two. The Control group changed by $37.25 \%$ in session one and only $25.31 \%$ in session two.

\section{Sympathetic activation pattern}

The SAP was not statistically significant between groups for treatment one $\left[\mathrm{F}(1,18)=1.330, p=.264\right.$, partial $\left.\eta_{2}=.069\right]$ but was a statistically significant over time groups $[\mathrm{F}(1,18)=255.025, p<$ .002 , partial $\left.\eta_{2}=.409\right]$. The SAP was statistically significant between the interventions and time in treatment two $[\mathrm{F}(1,18)=17.521, p$ $=.049$, partial $\left.\eta_{2}=.267\right]$. There was also a statistically significant change between groups $\left[\mathrm{F}(1,18)=4.042, p<.004\right.$, partial $\eta_{2}=$ .493]. 


\section{Discussion}

Biometric measurements (MAP, Pulse, HRV) taken in this study do not provide enough information to state conclusively the effects of Sensory Flow on the ANS. While all three show trends towards significance after the second Sensory Flow Treatment, none of the three achieve the desired level of significance. While statistical analysis did not reveal significant changes in some of the variables between groups, changes across time were seen in MAP during both sessions $\left\{\left[\mathrm{F}(3,54)=1.679, p=.003\right.\right.$, partial $\left.\eta_{2}=.578\right]$, [F (3,

54) $=4.335 p=.028$, partial $\left.\left.\eta_{2}=.423\right]\right\}$ and for pulse during session two $\left[F(3,54)=4.335 p=.028\right.$, partial $\left.\eta_{2}=.423\right]$. Significance here indicates that both the treatment and the control produced reduction in MAP and pulse, and therefore were both effective ways cause changes to these dependent variables. The effect size in both of these changes is interesting. While the intervention did not seem to make much of a change, both groups showed medium effect size, even with such a small N. The change in MAP and Pulse may be related to the effects of lying comfortably in a quiet room for a period of 15 minutes [42,43]. There was also an interesting trend between treatments one and two of Sensory Flow. It appears that treatment two produced improvements in all independent variables. (Figure 1) Although the effects are not considered significant, treatment two seems to be more effective than treatment one. The effect noted could be due to anticipation of the relaxation component of the treatment. Because patients consider the treatment results pleasant, they look forward to their effects, and thus prime the body for a better response. A study by Bishop MD, et al. [44] indicated a significant relationship between patient expectations and outcomes.

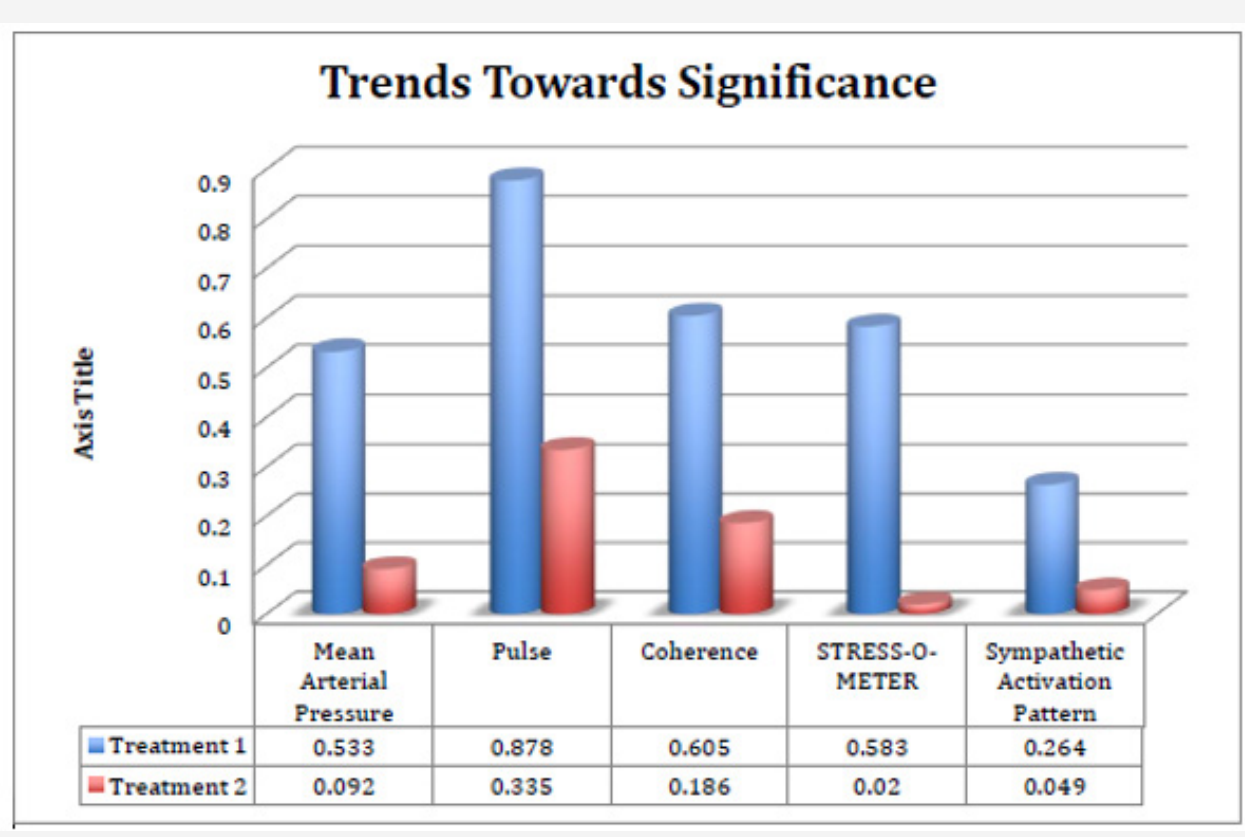

Figure 1: Changes in significance from treatments one to tswo of the Sensory Flow group.

In their study more than $80 \%$ of patients surveyed indicated that they expected that manual therapy would provide relief, and $87 \%$ felt that massage would significantly improve their pain [44]. Using massage to treat the patient did indeed provide better outcomes showing that a provider can expect better outcomes when they leverage patient expectations $[44,45]$. The trends towards significance could also be explained by the Polyvagal theory. According to the Polyvagal theory the social engagement role of the Vagus nerve determines how the ANS responds and reacts to the persons perceived environment [26]. Because the participant was exposed to the environment in the exact same manor in treatment one as in treatment two, the Vagus nerve may have been less engaged in the social engagement aspect of the intervention, and is thus more free to carry out the role of the Vagal brake $[26,46]$.

Limitations of This Study Include Not Being Able to Constantly Monitor Blood pressure Parati G, et al. [47] and having a small sample size. It is also possible that the lack of significance is due to a Type II error and that simply having a larger $\mathrm{N}$ would affect the outcomes. It does appear that the effects of Sensory Flow may build over time. Used with the other components of the AAT system such as Reflexercise and Congruence, additional benefits are likely to be seen [23]. Further research on the cumulative effects of Sensory Flow, and the AAT system as a whole are needed to make any conclusive Statements [48-53].

The positive results of this study do support the use of Sensory Flow as a mechanism to help decrease perceived stress, and to help decrease sensitivity to pain in patients who are up regulated. Both measures showed MCID's on the NRS, meaning that tenderness to palpation and stress both improved enough to be considered important to patients. Decreasing perceived stress and sensitivity to pain could interrupt faulty pain cycles, freeing the patient to deal with only the pain that is being caused by a present condition $[18,19]$. 


\section{Conclusion}

While more research is necessary to confirm the results of this study, and to further determine both the cumulative effects of Sensory Flow as a stand-alone treatment. Sensory Flow does seem to provide a gateway to decrease stress and perceived pain in participants who were known to be in an up-regulated state. Sensory Flow could then be a helpful component of beginning to treat patients with chronic pain.

\section{Acknowledgement}

This research did not receive any specific grant from funding agencies in the public, commercial or not-for-profit sectors.

\section{Conflict of Interest}

No conflict of interest.

\section{References}

1. Lang PJ, Bradley MM, Cuthbert BN (1990) Emotion, attention, and the startle reflex. Psychol Rev 97(3): 377-395.

2. Scaer RC (2001) The neurophysiology of dissociation and chronic disease. Appl Psychophysiol Biofeedback 26(1): 73-91.

3. Scaer DR (2011) Trauma, The Freeze Response and It's Clinical Syndromes: The foundational principals for the treatment of chronic pain. Wellness and Performance.

4. Van der Kolk B (2014) The Body Keeps the Score: Brian, Mind and Body in the Healing of Trauma. Penguin Group, USA

5. Van der Kolk B (1994) The body keeps the score: Memory and the evolving psychobiology of posttraumatic stress. Harv Rev Psychiatry 1(5): 253-265.

6. Deyoung CG (2010) Discussion on "Automatic and Controlled Processes in Behavioural Control: Implications for Personality Psychology" by Corr Mapping Personality Traits onto Brain Systems: BIS, BAS FFFS and Beyond. Eur J Pers 422: 404-422.

7. Bracha HS (2004) Freeze, flight, fight, fright, faint: adaptationist perspectives on the acute stress response spectrum. CNS Spectr 9(9) 679-685.

8. Bracha HS (2004) Can premorbid episodes of diminished vagal tone be detected via histological markers in patients with PTSD? Int. Int J Psychophysiol 51(2): 127-133.

9. Hamilton L (1989) Fight, flight or freeze: Implications of the passive fear response for anxiety and depression. hobia Practice \& Research 2(1): 17-27.

10. Vaiva G, Brunet A, Lebigot F, Boss V, Ducrocq F, et al. (2003) Fright (effroi) and other peritraumatic responses after a serious motor vehicle accident: Prospective influence on acute PTSD development. Can Psychiatry 48(6): 395-401.

11. Haker E, Egekvist H, Bjerring P (2000) Effect of sensory stimulation (acupuncture) on sympathetic and parasympathetic activities in healthy subjects J Auton Nerv Syst 79(1): 52-59.

12. Tiller W, Mccraty R, Atkinson M (1996) Cardiac Cohrence: A new noninvasive measure of autonomic nervous system order. Altern Ther Health Med 2(1): 52-65.

13. Kolb B, Whishaw I (2011) An Introduction to Brain and Behavior. Worth Publishers, USA.

14. Malliani A, Pagani M, Lombardi F, Cerutti S (1991) Cardiovascular neural regulation explored in the frequency domain. Circulation 84(2): 482 492.

15. Berntson G (1997) Heart Rate Variability: Origins, methods and interpretative caveats. Psychophysiology 34(6): 623-648.
16. Eppinger H, Hess L, Kraus WM, Jelliffe SE (1917) Vagotonia: a clinical study in vegetative neurology, Journal of nervous and mental disease. The Nervous and Mental Disease Publishing Company :116.

17. Julius S (1991) Autonomic nervous system dysregulation in human hypertension. Am J Cardiol 67(7): 3B-7B.

18. Schlereth T, Birklein F (2008) The Sympathetic Nervous System in Pain. Neuromolecular Med 10(3): 141-147.

19. Chapman CR, Tuckett RP, Song CW (2008) Pain and stress in a systems perspective: reciprocal neural, endocrine, and immune interactions J Pain 9(2): 122-145.

20. Melzack R (1999) Pain--an overview. Acta Anaesthesiol Scand 43(9): 880-884.

21. Melzack R (2001) Pain and the Neuromatrix In the Brain. J Dent Educ 65(2): 1378-1382.

22. Porges SW (1992) Vagal tone: a physiologic marker of stress vulnerability. Pediatrics 90 (3 pt 2): 498-504.

23. Musgrave S (2011) AAT for Professionals: Level 1 Online Course.

24. Nathan PW (1983) Pain and the sympathetic system. J. Auton. Nerv. Syst $7(3): 363-370$.

25. Craig AD (2003) Pain mechanisms: labeled lines versus convergence in central processing. Annu Rev Neurosci 26: 1-30.

26. Porges SW (2001) The polyvagal theory: phylogenetic substrates of a social nervous system. Int J Psychophysiol 42(2): 123-146.

27. Porges SW (2009) The polyvagal theory: New insights into adaptive reactions of the autonomic nervous system. Cleve Clin J Med 76: S86-S90.

28. Mercuri LG, Olson RE, Laskin DM (1979) The Specificity of Response to Experimental Stress in Patients with Myofascial Pain Dysfunction Syndrome. J Dent Res 58(9): 1866-1871.

29. Schwartz GE, (1972) Voluntary Control of Human Cardiovascular Integration and Differentiation through Feedback and Reward. Science 175: 90-93.

30. Sztajzel J (2004) Heartrate variability: a noninvasive electrocardiographic method to measure the autonomic nervous system. Swiss Med Wkly 134(35-36): 514-522.

31. Holmes T, Rahe R (1967) The Social Readjustment Rating Scale. J. Psychosom. Res11: 213-218.

32. Henry JD, Crawford JR (2005) The short-form version of the Depression Anxiety Stress Scales (DASS-21): construct validity and normative data in a large non-clinical sample. Br J Clin Psychol 44(2): 227-239.

33. Daniels JW, Molé PA, Shaffrath JD, Stebbins CL, Mahmud A (1998) Effects of caffeine on blood pressure, heart rate, and forearm blood flow during dynamic leg exercise during exercise J Appl Physiol (1985) 85(1): 154159.

34. Altunkan S, Iliman N, Kayaturk N, Altunkan E (2007) Validation of the Omron M6 (HEM- 7001-E) upper-arm blood pressure measuring device according to the International Protocol in adults and obese adults Blood Press Monit 12(4): 219-225.

35. Veiga OL, Gómez Martínez S, Martínez Gómez D, Villagra A, Calle ME, et al. (2009) Physical activity as a preventive measure against overweight, obesity, infections, allergies and cardiovascular disease risk factors in adolescents: AFINOS Study 46 protocol. BMC Public Health 475(9).

36. Shahidi B, Haight A, Maluf K (2013) Differential effects of mental concentration and acute psychosocial stress on cervical muscle activity and posture. J Electromyogr Kinesiol 23(5): 1082-1089.

37. Sonnesen L, Svensson P (2013) Jaw-motor effects of experimental jawmuscle pain and stress in patients with deep bite and matched control subjects. Arch Oral Biol 58(10): 1491-1497.

38. Fischer AA (1987) Pressure algometry over normal muscles. Standard values, validity and reproducibility of pressure threshold. Pain 30(1): 115-126. 
39. Cohen J (1992) A power primer. Psychol Bull 112(1): 155-159.

40. (2004) The Seventh Report of the Joint National Committee on Prevention, Detection, Evaluation, and Treatment of High Blood Pressure. Hypertension 42(6): 1206-1252.

41. Farrar JT, Young JP, La Moreaux L, Werth JL, Poole, RM (2001) Clinical importance of changes in chronic pain intensity measured on an 11-point numerical pain rating scale. Pain 94(2): 149-158.

42. Bahrke MS, Morgan WP (1978) Anxiety reduction following exercise and meditation. Cognitive Therapy and Research 2(4): 323-333.

43. Morgan WP (1979) Anxiety Reduction Following Acute Physical Activity Psychiatric Annals 9(3): 36-45.

44. Bishop MD, Mintken PE, Bialosky JE, Cleland JA (2013) Patient expectations of benefit from interventions for neck pain and resulting influence on outcomes. J Orthop Sports Phys Ther 43(7): 457-65.

45. Benz LN, Flynn TW (2013) Placebo, nocebo, and expectations: Leveraging positive outcomes. J Orthop Sports Phys Ther 43(7): 439-441.

46. Porges SW (2007) The Polyvagal Perspective. Biol Psychol 74(2): 116143.
47. Parati G, Casadei R, Groppelli A, Di Rienzo M, Mancia G (1989) Comparison of fingerand intra-arterial blood pressure monitoring at rest and during laboratory testing. Hypertension 13(6 pt 1): 647-655.

48. Williamson A, Hoggart B (2005) Pain: a review of three commonly used pain rating scales. J Clin Nurs 14(7): 798-804.

49. Tarvainen MP, Niskanen JP, Lipponen JA, Ranta Aho PO, Karjalainen Pa (2014) Kubios HRV--heart rate variability analysis software. Comput. Methods Programs Biomed 113(1): 210-220.

50. Crawford JR, Henry JD (2003) The Depression Anxiety Stress Scales (DASS): normative data and latent structure in a large non-clinical sample. Br J Clin Psychol 42(2): 111-131.

51. Simms RW, Goldenberg DL, Felson DT, Mason JH (1988) Tenderness In 75 Anatomic Sites. Arthritis Rheum 31(2): 182-187.

52. Schenk P, Laeubli T, Klipstein A (2007) Validity of pressure pain thresholds in female workers with and without recurrent low back pain. Eur Spine J 16(2): 267-275.

53. Cunha CO, Pinto Fiamengui LMS, Castro a CPC, Lauris JRP, Conti PCR (2014) Determination of a pressure pain threshold cut-off value for the diagnosis of temporomandibular joint arthralgia. J Oral Rehabil 41(5): 323-9. 